\title{
Power based event detection for electrical appliances
}

$$
\text { Xiu-wei WANG }{ }^{1, a} \text {, Yan-ping Cong }{ }^{2, b}, \text { Bo YIN }{ }^{3 *, c}
$$

\author{
1,2,3 Ocean University of China, No.238, Songling Road, Laoshan District, Qingdao City, Shandong \\ Province, China
}

a17864270587@sina.cn, ${ }^{b}$ congyp@ouc.edu.cn, cybfirst@126.com

\begin{abstract}
Keywords: Non-Intrusive Appliance;Event Detection;Goodness-of-Fit;Generalised Likelihood Ratio Test;Power different
\end{abstract}

\begin{abstract}
Appliance specific energy monitoring is perceived as a prerequisite for reducing energy usage in households.In this paper, a non intrusive monitoring method is introduced, and a power identification algorithm based on transient detection is introduced. Then the simulation experiment is carried out by using different analysis methods. In order to improve the accuracy of the opening and closing of smart grid and adapt to the development trend of recognition of household appliances, based on this algorithm is put forward in the circuit current, power and other parameters combined with information, if the average power of several cycles of adjacent difference changes different from the baseline, a fixed threshold is exceeded. This event will be recorded, and based on the MATLAB platform, the household appliances simulation, test data, the algorithm was verified, the results show that the proposed method to detect electric power has a good recognition results.
\end{abstract}

\section{Introduction}

Reducing energy consumption is an imperative, nationally, commercially, and individually. However, energy conservation is challenging, one of the major reasons being consumers' lack of awareness about energy usage patterns [1].As a first step towards energy conservation and emission reduction, in terms of energy use, a higher transparency in place of the traditional hidden nature of the trend must be generated.As a first step towards energy conservation and emission reduction, in terms of energy use, a higher transparency in place of the traditional hidden nature of the trend must be generated. With the improvement of people's living standard, household consumption is also growing, which increases the peak valley difference, cause a lot of waste of energy, has a great impact on the power grid. Then, the household electricity management becomes very important. The household electricity monitoring system not only can solve the situation of electricity users on their understanding of the problem, it is very important to reduce the energy consumption has the significance[2,3].

Non intrusive electrical load monitoring (NILM) is considered to be the most promising and scalable solution for obtaining home appliance specific energy information. For household appliances power consumption monitoring in two ways: one is invasive monitoring method[4], the installation of voltage and current sensor in power supply port of each household appliance, the disadvantage is the high price, complex installation; the other is a non-invasive method for monitoring [6], the voltage and current sensors installed in the client only the user, real-time acquisition of the voltage and current data were analyzed by software, to determine the electricity consumption of household appliances[7]. Non invasive and invasive method of monitoring electrical appliance monitoring methods, the former investment cost is low, and the monitoring device can continuously capture every moment of load information, it is suitable for online applications.

With the rapid development of the electronics industry and software industry, The authors in $[8,9]$,propose the software and hardware realization of power load monitoring system based on embedded processor, but did not propose specific electricity monitoring method. At present, the United States and Japan are in the non intrusive online monitoring research, domestic research in this area is very little, is in the theoretical experimental stage. In this paper, we use the power to detect the switching event of a household appliance. In general, an event detector algorithm is used to find the variation of the power signal between the two stable states. According to the power difference 
between adjacent cycles, and based on the MATLAB platform, if the difference exceeds the specified threshold, the electrical switch event will be marked out. The simulation results show that this method has a better recognition effect.

\section{Event Detection Algorithm and Power Calculation}

The measurement of electric energy parameters is the basis of calculating the power consumption of household electrical appliances. If one of the power parameter errors is too large, it will lead to an error in the type of electrical appliances. In this paper, voltage and current sensors to accurately measure the voltage and current, and through the MATLAB platform to calculate the power of a variety of electrical. Some statistical based methods have been proposed to identify these electrical switching events.Of those, Generalised Likelihood Ratio Test (GLR) and mean-difference based methods are frequently used. These methods are mostly applied in an offline based manner, i.e. passing the whole data stream to the algorithm.It is different from the online event detection algorithm, in which the power measurement data is introduced into the algorithm by one by one. The algorithm proposed in this paper is based on the power difference of the different periods, if a power difference (in the observed sample) is different from the baseline, beyond a fixed threshold, this event will be recorded.

\section{Event detection}

A Experimental platform to build. The identification technology based on the power change is to judge a switch condition of an electrical appliance by using the variation of the consumption data of the whole power consumption. Power algorithm through the device load curve, to detect a device to open and close. Sometimes, different appliances will produce the same results (such as a 150W computer and a 150W TV), and they are only different in time. Details of the experimental platform to build as shown in fig.1.

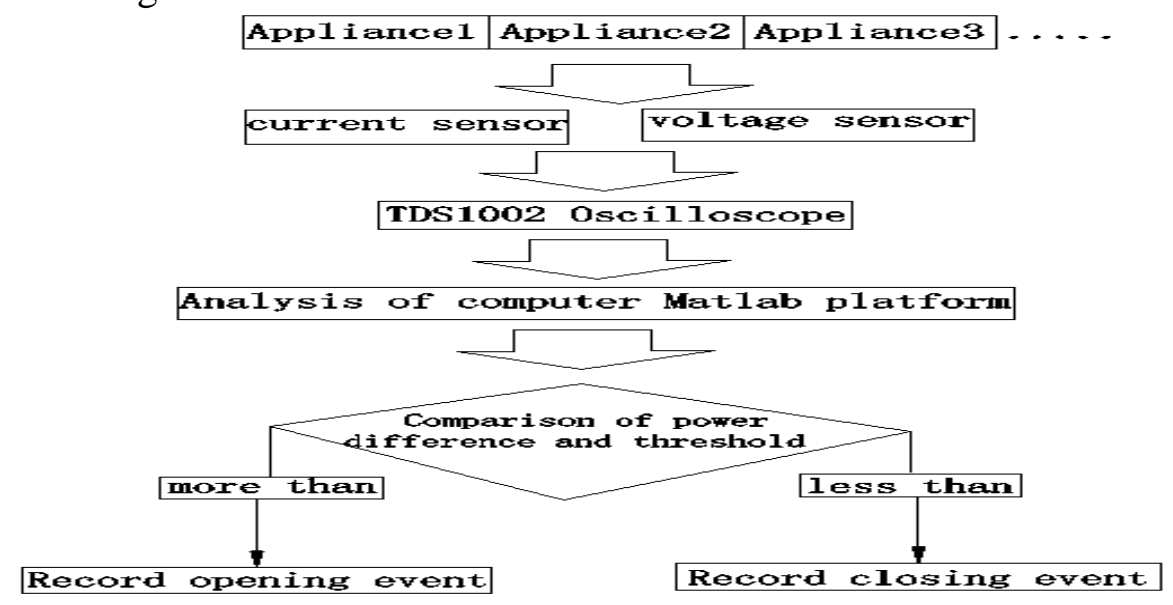

Fig.1. Block diagram of experimental platform design

B Experimental Principle. If a power change (in the observed sample) is different from the baseline, beyond a fixed threshold, the event will be recorded. The algorithm needs to set two parameters: the number of samples per cycle and a power threshold. The working principle of the algorithm is as follows: with the passage of time and the received energy signal data point is $\mathrm{N}$, set the power data points for each cycle is 74 . Then for each cycle the average value of the power, the power of the average value of the adjacent cycle (a cycle by cycle) and the subtraction, difference and threshold comparison. Returns a decision vector containing TRUE or FALSE values (if the difference exceeds a given threshold value of ON, or OFF). If the result of the value of the decision vector is $\mathrm{ON}$, and the set of 50 cycles is no longer repeated detection, then an open event is detected. If the result is OFF, and the 10 cycle is no longer detected open events, then said a shutdown event was detected. Information about event location and average power difference is stored in the event queue. The location of the event is eleventh cycles after the start of the judge and record. 
$\Delta P_{\text {different }}=\left(P_{\text {different }}\right)_{s+1}-\left(P_{\text {different }}\right)_{s}$

$P_{\text {different }}=\frac{\sum_{k=1}^{N} \mid p(k)-\text { mean }(p) \mid}{N}$

Fig. 2 is the difference in power of different power equipment in different periods. By the Eq.1 and Eq.2 the difference of power, through the power difference compared with the threshold, and set a certain period of time will not repeat event detection switch electrical appliances, power and waveform in MATLAB in calibration, the switch state in a moment of electrical appliances.

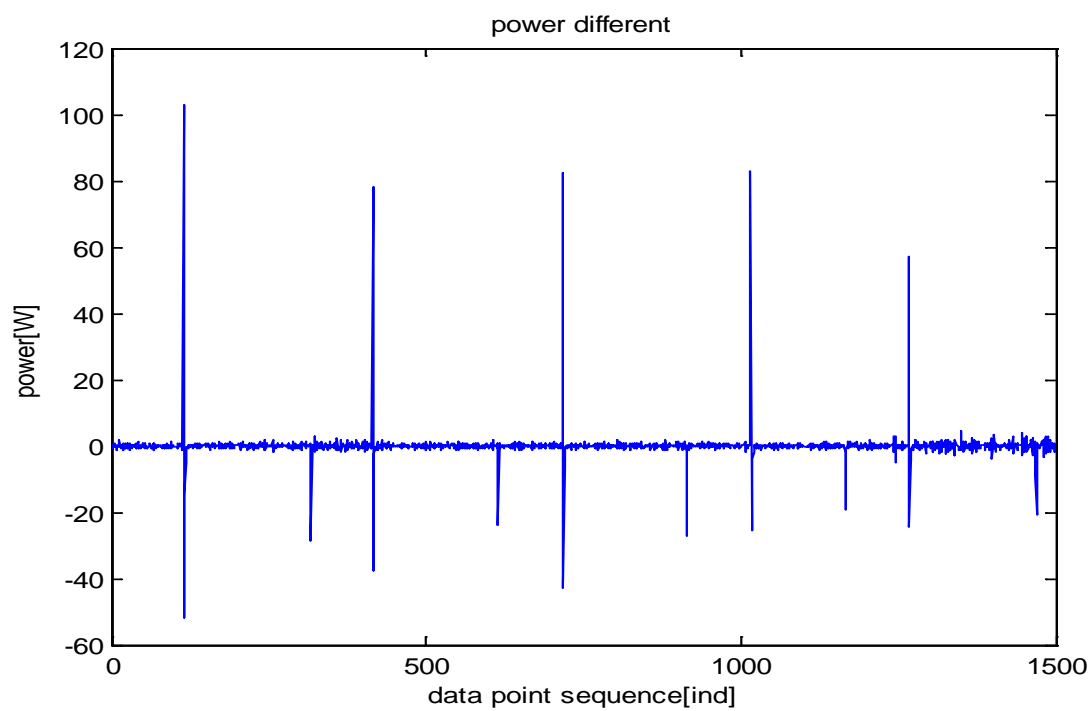

Fig.2. Power difference snapshots of different periods

\section{Experimental Process and Analysis}

From high power, medium power and small power to pick a typical household electrical appliances as a group of research objects, this paper select the common household appliances: air conditioning, fluorescent lamp, electric fan, computer as the research object. Fig.3 and Fig.4 is the transient power waveform of the 4 types of appliances at startup and shutdown.
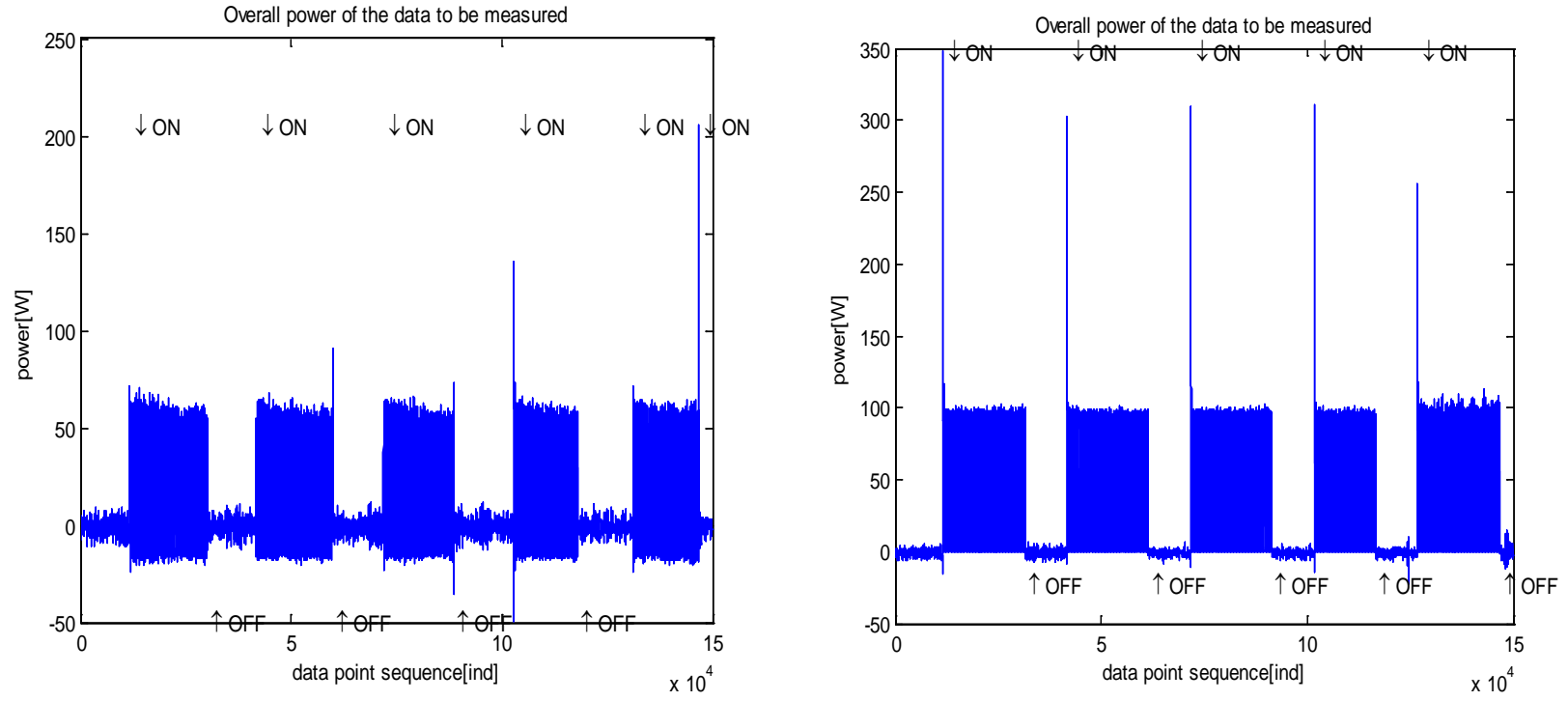

Fig.3. Transient waveform of starting and closing time of Fluorescent lamp and Electric fan 

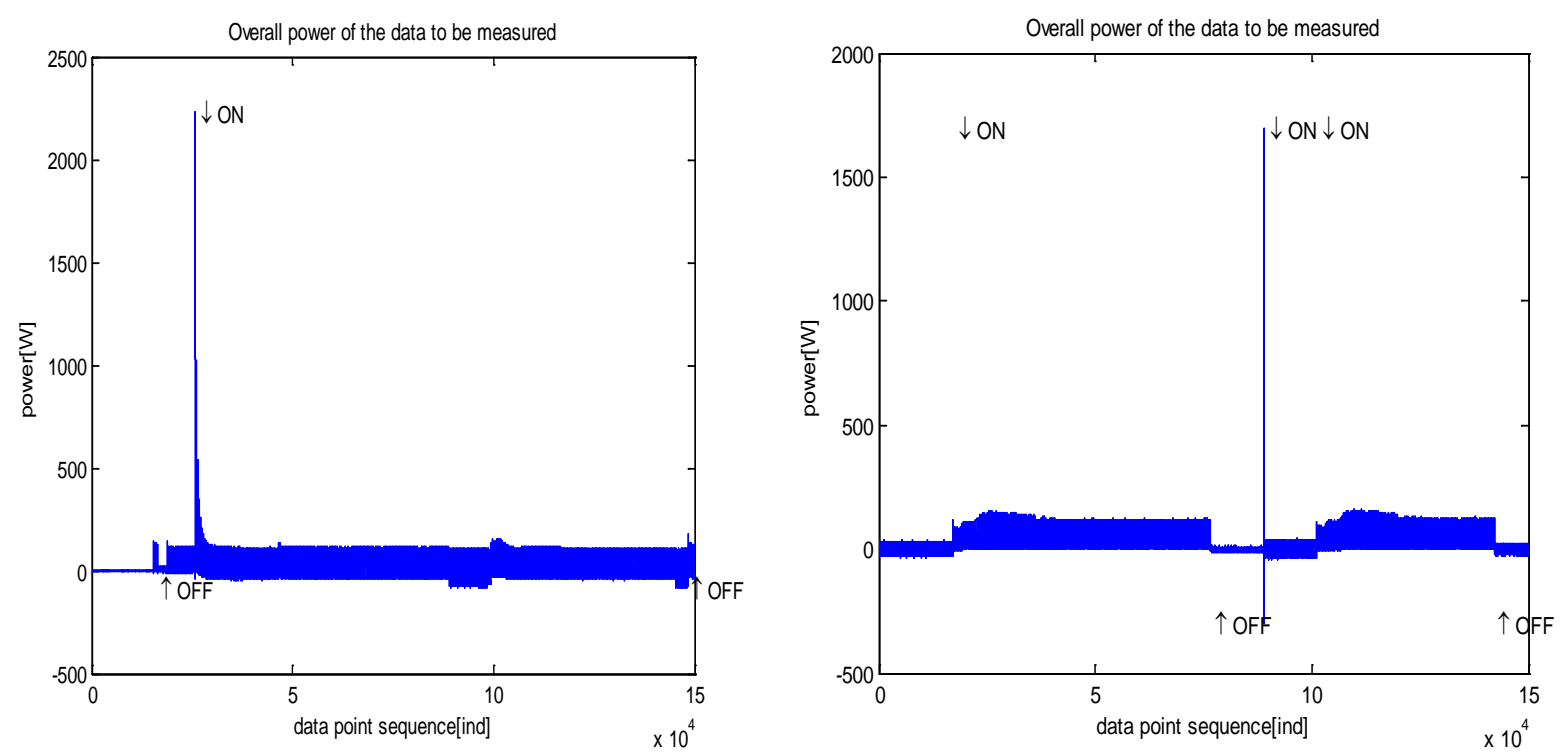

Fig.4. Transient waveform of starting and closing time of Air conditioner and Computer

After the voltage and the current sensor detects the voltage and current sampling data, and it is simulated in MATLAB, and then the data into the improved power difference algorithm to calculate the transient power waveform, the above four kinds of electrical appliances and their opening and closing events to determine the result. The results of the judgment are shown in table 1.

Table1. Determination result of electric switch event

\begin{tabular}{|c|c|c|c|c|}
\hline $\begin{array}{l}\text { Electrical } \\
\text { appliances }\end{array}$ & Opening times & Off times & accuracy rate & error \\
\hline Computer & 50 & 50 & $99 \%$ & $1.2 \%$ \\
\hline Fluorescent lamp & 50 & 50 & $100 \%$ & 0 \\
\hline Electric fan & 50 & 50 & $99 \%$ & $2.5 \%$ \\
\hline Air conditioner & 50 & 50 & $75 \%$ & $5.22 \%$ \\
\hline
\end{tabular}

the table 1, through the improved power difference method to determine the opening and closing of the event, the algorithm is verified, and the result of the switch event is obtained. It shows that the power difference event algorithm designed in this paper is suitable for the determination of electrical events.

\section{Summary}

In this paper, a power difference and threshold contrast algorithm based on transient detection is improved, and the model is built by using the simulation software MATLAB platform. By tracing the sampling data in the simulation software, four kinds of electric power transient waveform is obtained, and the judgment event marked in the graph of power. The results show that the improved algorithm can effectively identify different types of electrical equipment of power load in the opening and closing time, the electric equipment event detection accuracy can reach more than 95\%, show the superiority of the algorithm is powerful, but not be able to identify the different electrical monitoring of the same power, improved this is the place to work in the future.

\section{Acknowledgements}

This work was financially supported by International S\&T Cooperation Program of China (2015DF R10490). 


\section{Reference}

[1]Alasalmi, T., Suutala, J., Rning, J.: Real-time non-intrusive appliance load monitor - feedback system for single-point per appliance electricity usage. In: SmartGreens, pp. 203-208. SciTePress

[2]Anderson, K., Berges, M., Ocneanu, A., Benitez, D., Moura, J.: Event detectionfor non intrusive load monitoring. In: IECON 2012 - 38th Annual Conference on IEEE Industrial Electronics Society, pp. 3312-3317 (October 2012)

[3]Armel, C.K., Gupta, A., Shrimali, G., Albert, A.: Is disaggregation the holy grail of energy e ciency? the case of electricity. Energy Policy 52(C), 213-234 (2013)

[4]Berges, M., Goldman, E., Matthews, H., Soibelman, L., Anderson, K.:User-centered nonintrusive electricity load monitoring for residential buildings. Journal of Computing in Civil Engineering 25(6), 471-480 (2011)

[5]Fischer, C.: Feedback on household electricity consumption: A tool for saving energy? Energy E ciency 1, 79-104 (2008)

[6] C. Laughman, K. Lee, R. Cox, S. Shaw, S. Leeb, L. Norford,and P. Armstrong, "Power signature analysis,” IEEE Power and Energy Mag., pp. 56-63, March/April 2003.

[7]S. Shaw, S. Leeb, L. Norford, and P. Armstrong, "Nonintrusive load monitoring and diagnostic in power systems," IEEE Transactions on Instrumentation and Measurement,vol. 57, no. 7, pp. 1445-1454, July 2008.

[8]N. Vaswani, "The modified cusum algorithm for slow and drastic change detection in general hmms with unknown change parameters,” in ICASSP’05, vol. 4. IEEE, 2005,pp. 701-704.

[9]Kazmi, A.H., O’Grady, M.J., Delaney, D.T., Ruzzelli, A.G., O’Hare, G.M.P.: A review of wireless-sensor-network-enabled building energy management systems. 10(4), 66:1-66:43 (2014) 